\title{
Stage I Ileal Neuroendocrine Tumor AJCC v8
}

National Cancer Institute

\section{Source}

National Cancer Institute. Stage / Ileal Neuroendocrine Tumor A/CC v8. NCI Thesaurus. Code C135125.

Stage I includes: T1, N0, M0. T1: Tumor invading lamina propria or submucosa and measuring $1 \mathrm{~cm}$ or less in size. NO: No regional lymph node metastasis. M0: No distant metastasis. (AJCC 8th ed.) 